\section{Die Räume der Milieus}

\author{
Verkehrspolitik und -planung stehen dem Wachstum von Verkehrsaufwand und \\ Motorisierung bisher noch weitgehend machtlos gegenüber. Erforderlich ist \\ daher ein Perspektivwechsel der Stadt- und Mobilitätsforschung. Es heißt \\ Abschied zu nehmen von vereinfachten Annahmen über die Personenmobilität, \\ ebenso wie von eindimensionalen Bildern vom städtischen Raum und dessen \\ Wechselwirkungen mit dem Verkehr.
}

$\mathrm{D}$

Von Markus Hesse. ie empirische Stadt- und Verkehrsforschung versucht, die Bereiche Raumund Siedlungsentwicklung sowie Mobilität im Sinne einer , integrierten“ Planung zusammenzuführen bzw. die entsprechenden Voraussetzungen dafür zu schaffen (1). Im Zeichen der vielschichtigen, sich wechselseitig verstärkenden Probleme städtischer Entwicklung und des Verkehrs ist eine solche Integration wünschenswert. Es wäre auch schon viel gewonnen, würde sich das Ziel der Verkehrsabwicklung stärker als bisher anderen städtischen Nutzungen unterordnen. Doch bestehen Zweifel, ob eine verkehrssparsame Integration der Systemlogiken von Siedlungsentwicklung und räumlicher Mobilität konsequent gelingen kann - vom theoretischen Design her und in der Praxis.

So richtig die Vermutung ist, dass Mobilität und Verkehr auch durch räumliche Arrangements bestimmt sind, so wenig griffig scheinen die Spielräume, diesen Kontext steuern zu können. Auch wird bei der Suche nach Handlungsoptionen häufig übersehen, dass sich beide Elemente dieser Wirkungsbeziehung städtische Nutzungen und Verkehr - radikal verändern. Einer der „Kofferbegriffe“ für diese neuen Phänomene ist der des „Lebensstils": qualitativ schillernd, quantitativ kaum durchdrungen, schwer operationalisierbar. Gleichwohl spricht vieles dafür, dass es diese neuen Verhaltensmuster gibt und dass sie von Bedeutung für dieses Thema sind. Mit Hilfe einer räumlichen Lesart des in den Sozial- und Raumwissenschaften diskutierten MilieuBegriffs (siehe Kasten) soll hier der Versuch gemacht werden, zu einem tieferen Verständnis der relevanten Prozesse zu kommen (2). Ziel ist es, dem veränderten Bild von städtischen Nutzungen und Verkehr besser als bisher gerecht $\mathrm{zu}$ werden.
Stadtentwicklungsprozesse verlaufen in den meisten Industrieländern gegenwärtig mit einer erhöhten Entwicklungsdynamik. In räumlicher Hinsicht sind sie stark ausdifferenziert, wie die vermeintlich widersprüchliche Parallelität von Aufrüstung der Innenstädte, Maßstabssprung bei Handel und Entertainment oder selektiver Abwanderung andeutet. Unstrittig scheint zu sein, dass die stadträumliche Dynamik hybride, netzwerkartige Formen der Raumorganisation fördert, klare Strukturtypen (Stadt, Land) oder dominante Zentren dagegen an Bedeutung verlieren. Nicht zufällig haben intermediäre Begrifflichkeiten wie ,Zwischenstadt" Konjunktur (3).

\section{Dynamik der Stadträume}

Dieser stadträumliche Wandel, den man auch als Ausdruck umfassender Flexibilisierung und Fragmentierung ansehen kann, prägt zunehmend den Rahmen der Mobilitäts- und Verkehrsentwicklung. In dieser Gemengelage wird das räumliche Beziehungsgefiuge stark verändert; vielfach werden kleinräumige Beziehungen durch grobmaschige Netze ersetzt, mit Zunahme der Distanzen und steigender Komplexität der Wegeketten ver-

\section{Lebensstile und Milieus}

Hradil begreift Lebensstile als "typische Verhaltensmuster sozialer Gruppierungen", die zwischen den strukturellen Bedingungen des menschlichen Handelns und dem praktischen Handeln selbst angesiedelt sind. Dem Begriff des Milieus kommt seines Erachtens eine Filterfunktion zu, er dient zur Kennzeichnung von Gruppen von Menschen, die "üußere Lebensbedingungen und/oder innere Haltungen aufweisen, aus denen sich gemeinsame Lebensstile herausbilden." (4) Matthiesen sieht die Funktion sozial-räumlicher Milieus zwischen Makrotrends und lokalen Entwicklungsverläufen darüber hinaus als Katalysatoren und Vermittler, als einbettende Strukturen für Raumplanungen, schließlich als Impulsgeber für innovative Selbstorganisationsprozesse (5). ändern sich Raum-Zeit-Muster und Verkehrsmittelwahl erheblich. Bisher wird dieser Befund nicht angemessen wahrgenommen respektive systematisch untersucht. In den Köpfen der Stadtund Verkehrsplaner dominiert noch das Bild der kompakten, ,europäischen“ Stadt als Referenzrahmen für den Sollzustand. Es dient auch als Ausgangsbasis eines großen Teils der Empirie. Damit ist der analytisch-methodische Zugang zum Problem in mehrfacher Hinsicht unvollständig. Hinzu kommt, dass sich der skizzierte Wandel auch auf einer symbolischen Ebene materialisiert, deren alltagspraktische Relevanz häufig unterschätzt wird. Dies zeigen drei Beispiele aus aktuellen Verlaufsformen von Transformationsprozessen in 0stdeutschland:

- Die neuen Gewerbegebiete an den Rändern der Städte signalisieren die ungebrochene Vitalität der „Ökonomie des Autos“. Die baulichräumliche Dominanz von Tankstellen, Autowaschanlagen, Kfz-Handel, -Werkstatt und Teilehandel ist nicht nur real, sondern auch imageverfestigend - pro Automobil.

- Das Einkaufszentrum (,Shopping Mall“) bildet zunehmend den ökonomischen und kulturellen Kristallisationspunkt eines konsum- und mobilitätsgeprägten Lebens. Dieses organisiert sich in hohem Maße uiber den Pkw-Verkehr.

- Die Tankstelle dient nicht mehr nur zum Auftanken von Pkw, sondern hat zunehmend auch sozialräumliche Funktionen. Sie ersetzt vielerorts den Einzelhandel, ist Treffpunkt von Subkulturen, Autonarren und Skinheads.

Diese Beispiele symbolisieren einen tiefgreifenden Wandel unserer Lebenswelt, hinter dem komplexe Raum-Zeit-Muster und Raumwahrnehmungen stehen. Sie sind durch die Überlagerung unterschiedlicher „Be-Deutungen“ von Raum und Raumüberwindung geprägt. Zur theoretischen Analyse dieser mehrdimensionalen „Räumlichkeiten“ gibt es sehr verschiedene Ansätze (6). Sie sollten in der Mobilitätsforschung mehr Beachtung finden. Möglicherweise bauen diese „symbolischen“ Räume auch eine Brïcke von der Raum-Zeit-Struktur zum individuellen Handeln. Dieser Übergang wird hier mit dem Begriff des „Milieus“ versucht.

\section{Sozialer Wandel und Milieus}

Der Begriff des Milieus steht für die am konkreten Ort sich uiberlagernden Erfahrungs-, Deutungsund Handlungsmuster. Ausgangspunkt ist hier die Annahme, dass das Verkehrswachstum nicht allein mit der Expansion der Räume oder der Auswei- 
tung des Verkehrssystems erklärbar ist. Dafür fallen die individuellen und sozialen Brüche, die hier eine Rolle spielen, zu umfangreich aus. „Milieu“ meint die Entwicklung sozialer Beziehungssysteme unter den Bedingungen von Ausdifferenzierung und Wählbarkeit. Parallel zu der Annahme, dass soziale Aktivitäten immer weniger räumlich determiniert sind, werden gleichzeitig neue Formen der gegenseitigen Durchdringung von räumlichen Arrangements (Identitäten, Erreichbarkeiten, territoriale Konflikte) und sozialem Handeln vorgefunden. Milieus sind auch räumlich bedingt, wenngleich längst nicht mehr an bestimmte Räume gebunden. Schulze nennt unterschiedliche Bedeutungen von Raum: Umgebung (Territorium), Szenerie (Treffpunkte und Schauplätze der Selbstdarstellung) und milieuneutraler Raum (übrige Areale) (7).

Der soziale Wandel demonstriert die unterschiedlichsten Milieukonstellationen und deren Raumbezüge. Individuelle Handlungsmuster haben sich im Zeichen höherer Einkommen auf der einen und von Erwerbslosigkeit auf der anderen Seite (also bei wachsender sozialer Polarisierung) sowie durch neue Arbeitszeiten und veränderte Freizeitmuster stark gewandelt. Das Spektrum beruflicher und privater Tätigkeiten hat sich erweitert. Persönliche Aktionsräume werden ausgedehnt. Selbst am unteren Ende der Wohlstandsskala, bei Marginalisierten und Deprivilegierten in Städten, steigt der Bedarf an Beweglichkeit und Erreichbarkeit. Gleiches gilt für die Fälle, wo flexible Arbeit an mehreren Teilzeit-Orten an die Stelle der früher standorttreuen Fabrikarbeit tritt. Diese Grundprobleme räumlicher Fragmentierung sind natürlich nicht per se lösbar. Auf den ersten Blick verliert der Raum in diesen Zusammenhängen dramatisch an Bedeutung, der Stellenwert räumlicher Mobilität steigt. $\mathrm{Ob}$ dies als einheitliches Muster zwangsläufig auftritt, ist aber mindestens umstritten, wenn es nicht sogar gegenläufige Tendenzen gibt.

\section{- Neuer Forschungsansatz}

Zunächst soll diese Entwicklung nicht auf Interventionsmöglichkeiten hin diskutiert werden, sondern mit Blick auf einen geeigneten Zugang der Forschung. Der methodologische Clou der Milieu- und Lebensstildebatte könnte in der Verschränkung individueller Handlungspraktiken und Stilisierungen auf der einen und sozial-räumlicher Arrangements auf der anderen Seite liegen. Damit könnte mehr Licht in die wechselseitige Bedingtheit von stadträumlicher Struktur und
Mobilität gebracht werden - ohne den vagabundierenden Großparadigmen von Globalisierung oder Individualisierung ungeprïft eine $\mathrm{zu}$ hohe alltags- und lebensweltliche Prägekraft zuzubilligen. Gleichzeitig ist dieser Ansatz hinreichend offen, um der deterministischen Sackgasse kausaler Wirkungsbeziehungen zu entgehen.

Meines Erachtens ist es nicht nur geboten, Mobilität und Verkehr, über klassische soziodemografische Determinanten hinaus, möglichst differenziert und zeitgemäß zu analysieren. Dieser Versuch allein wäre zwar schon verdienstvoll, ist aber wissenschaftlich nicht mehr ganz originell. Mich interessiert hier, ob und inwieweit diese Analyse mit typischen Mustern räumlicher Vergesellschaftung einher geht. Damit ist die Hypothese verbunden, dass es einen Bedeutungswandel in der räumlichen Konfiguration von Mobilität und Verkehr gibt, der nur auf den ersten Blick eine schwache Wirkungsbeziehung zwischen Raum und Milieu widerspiegelt. Bei näherer Betrachtung ergeben sich gemeinsame Kontexte, aus denen (Verkehrs-) Entstehungsbedingungen und (städtische) Wirkungsfelder ablesbar sind; diese Kontexte wurden in der Forschung bisher jedoch stark vernachlässigt.

Die Grundthese liest sich wie folgt: Die sich stark verändernden städtischen Räume sind

a) weit heterogener strukturiert als bisher unterstellt,

b) mit verschiedenen Deutungen ,aufgeladen“ und

c) üben einen - gleichwohl schwer bestimmbaren - Einfluss auf Mobilität und Verkehr aus. Heute werden sie zunehmend sozial gefiillt und die Bedeutung von Räumen, in denen sich spezifische soziale Arrangements niederschlagen, steigt. In der lebensstilbezogenen Forschung wurde versucht, diesem Phänomen mit der Konstruktion typischer „Mobilitätsstile" zu begegnen (8). Deren Greifbarkeit ist aber im Licht des skizzierten Wandels eigentlich ein Widerspruch in sich: vermutlich werden mobile Lebensstile durch die gleichen Individuen sehr variabel ausgeübt (gewählt!).

Spannend wäre es $\mathrm{zu}$ klären, an welche physischen, sozialen oder in Szene gesetzten Räume Mobilität gekoppelt ist und inwieweit diese durch spezifische Milieus - im Sinne einer Überlagerung von Erfahrungs-, Deutungs- und Handlungsmustern - geprägt sind. In der sozialwissenschaftlichen Forschung besteht Einigkeit darïber, dass es solche Interaktionen von Raum und Subjekt gibt; die Frage ist, ob dies nicht auch für die Mobilitätsforschung nutzbar gemacht werden kann.

\section{Zwei Beispiele}

Paradebeispiel für die skizzierte Entwicklung ist die Gentrifizierung attraktiver Innenstadtquartiere durch urbane Milieus, deren räumliche Mobilität zumindest zu einem bestimmten Teil quartiersgebunden ist und in denen sich das stadträumliche und ökonomische Umfeld auch den Bedürfnissen dieser Milieus sichtbar angepasst hat. Komplementär dazu sind seine Protagonisten bekanntlich aber auch sehr fernireisefreudig, was die Ambivalenz dieser Entwicklung aufzeigt. Um ein anderes Beispiel zu nennen, fuir das die Verkehrsforschung bisher im Unterschied zur Stadtforschung wenig Interesse aufgebracht hat: Die informelle Ökonomie großer Städte bietet ein reiches Überlebensreservoir für diejenigen, die auf dem segmentierten Markt der hochqualifizierten Erwerbsarbeit kaum Chancen haben. Beschäftigte in Niedriglohnsektoren (etwa haushaltsnahe Dienste), andere geringfuigig Beschäftigte oder Saisonarbeiter gehören in diese Gruppe, aber auch Migranten und Illegale. Sie bilden nicht nur Milieus, sondern auch ,informelle Räume“, in denen sie sich physisch und sozial bewegen. Am Beispiel von zwei Quartieren in Brüssel wurden die Räume dieser informellen Ökonomie beschrieben, die einen starken Kontrast zu den suburbanisierten Mittelklasse- und Wohlstandsmilieus der belgischen Hauptstadt bilden (9). Es ist anzunehmen, dass es sich hier um typische Milieukonstellationen handelt, die auch mit spezifischen Mobilitätsmustern einhergehen. Vermutlich bringen diese an Bedeutung gewinnenden Facetten der städtischen Ökonomie ein anderes, konflikthaftes Muster räumlicher Mobilität hervor, als es unseren landläufigen Vorstellungen von Berufsverkehr entspricht. Milieuansätze könnten einen Beitrag zum besseren Verständnis der Raum-Zeit-Strukturen dieser Gruppen leisten.

Sozial-ökologiscbe
Unternebmensbewertung
ist das Thema
des Informationsdienstes
Ökologisches
Wirtschaften $2 / 2000$
Wenn Sie potenzielle Beiträge
haben, wenden Sie sich bitte
an die Redaktion.




\section{Ausblick}

Es geht an dieser Stelle nicht allein um akademische Fragen, um Forschungsmethoden, Theorien, Zugänge. Dahinter stehen ganz praktische Probleme:

a) die notwendige Erweiterung des Blickwinkels der Forschung auf die Wechselbeziehungen von Verkehr und Umfeld,

b) die im Sinne der forschungspraktischen Rationalisierung bisher überstrapazierte Reduktion dieses Gegenstandes.

Der ,politische" Charakter dieser Diskussion macht sich an zentralen Bewertungsfragen fest: Wie ist der Forschungsgegenstand aufgebaut, welche Einflüsse gehen auf externe und regionale Rahmenbedingungen zurück, wie stark sind Handlungsmuster individuell geprägt? Und: Welche Spielräume bleiben zur Gestaltung? Hier kann die Forschung Erkenntnisse, Positionen und Fragen beisteuern - nicht mehr, nicht weniger. Ein Ausweg aus dem bisherigen Dilemma zwischen Mikro- und Makroebene liegt womöglich in der Klärung des Zwischenraums (sic!): nämlich der regionalen Spezifik des Zusammentreffens von globalen bzw. externen Trends und lokalen oder regionalen Praktiken.

Forschungs- und planungspolitisch betrachtet gibt es aber nichts umsonst: Der Preis, der für mehr Gegenstandsgemäßheit und wissenschaftliche Aufrichtigkeit gezahlt werden muss, liegt vorläufig im Verzicht auf Rezepte - auch wenn die Forschung von der Praxis zunehmend darum gebeten wird und diesbezüglich stark unter Verwertungsdruck gerät. Im Zeichen der wachsenden Stilisierungen des Alltagslebens ist es sicher naiv anzunehmen, man könne von der differenzierten Abbildung von Lebensstilen und daraus „abgeleiteten“ Merkmalen eines veränderten Mobilitätshandelns gleich auf passgenaue Bausteine eines Mobilitätsmanagements umschalten. Dies wäre Sozialtechnik auf gehobenem Niveau, mit relativ kurzer Halbwertszeit. Erst recht ergeben sich daraus noch keine neuen „Märkte“ für die Verkehrswirtschaft.

Um einer erneuten Ent-Täuschung derjenigen vorzubeugen, die nach Steuerungsinstrumenten mit Hebelwirkung suchen, sei auf den vorläufigen, forschungsstrategischen Charakter dieser Überlegungen hingewiesen. Damit ist noch kein Abgesang an die Politik verbunden: Steht der Erfolg der integrierten Siedlungs- und Verkehrsplanung noch aus, dann misst sich diese Aussage zunächst an deren formuliertem Anspruch. Dass dieser bisher nicht erreicht wurde, muss nicht notwendigerweise an der Strategie selbst liegen, sondern kann viele Ursachen haben. Im Grunde gibt es zu einer behutsamen, tendenziell auf Verkehrszähmung statt -durchsetzung gerichteten Politik und Planung noch keine vernünftige Alternative.

Ob ihre Mittel und Blickwinkel zeitgemäß sind, darf aber bezweifelt werden. Der Forschungsansatz, für den hier plädiert wird, steht komplementär dazu. Er soll künftig relevante Fragen aufwerfen, ohne die aktuellen Aufgaben der Konfliktlösung in der Praxis zu vernachlässigen. Anders ausgedrückt: Man stelle sich vor, der derzeitige Stand der Diskussion um eine nachhaltige Mobilität wäre umgesetzt, und der Verkehr wäre immer noch nicht nachhaltig. Spätestens dann brauchen wir ein neues Forschungsparadigma und neue methodische Zugänge.

\section{Anmerkungen}

(1) Vgl. stellvertretend Holz-Rau, C.: Integrierte Verkehrsplanung - die herausgeforderte Fachplanung. In: Informationen zur Raumentwicklung 7/8, 1996, S. 391-416.

(2) Vgl. zu raumbezogenen "Milieu“-Forschungen Matthiesen, U. (Hrsg.): Die Röume der Milieus. Berlin 1998; vgl. auch Keim, K.D.: Milieu und Moderne. Zum Gebrauch und Gehalt eines nachtraditionalen Milieubegriffs. In: Berliner Journal für Soziologie, H. 3, S. 387-399.

(3) Sieveris, T.: Zwischenstadt. Braunschweig u. a., 1997.

(4) Hradil, S.: Sozialstrukturanalyse in einer fortgeschrittenen Gesellschaft. Opladen 1987, S. 164.

(5) Matthiesen, U.: Milieuforschung: Raumentwicklungen zwischen Innovationen und disparitären Entwicklungsmustern. In: IRS aktuell 19, 1998, S. 1.

(6) Vgl. Läpple, D.: Gesellschaftszentriertes Raumkonzept. Zur Überwindung von physikalisch-mathematischen Raumauffassungen in der Gesellschaftsanalyse. In: Wentz, M. (Hrsg.): Stadt-Räume. Frankfurt/Main 1991, S. 35-46. (7.) Vgl. dazu Schulze, G.: Milieu und Raum. In: Noller, P. et.al.: Stadt-Welt. Über die Globalisierung städtischer Milieus. Frankfurt/Main 1994.

(8) Götz, K. (Hrsg.): Mobilitätsstile. Ein sozial-ökologischer Untersuchungsansatz. Forschungsverbund City-Mobil Stadtvertrögliche Mobilität, Bd. 7, Frankfurt/Main 1998. (9) Kestelot, C./ H. Meert: Informal Spaces: The Geography of Informal Economic Activities in Brussels. In: International Journal of Urban and Regional Research (23), H. 2 , S. $232-251$

\section{Der Autor}

Dr. Markus Hesse ist wissenschaftlicher Mitarbeiter am Institut für Regionalentwicklung und Strukturplanung (IRS).

Kontakt: IRS, Flakenstr. 28-31, 15537 Erkner.

Tel. 03362/ 793-157, Fax - 111,

E-muil: hessem@irs.los.shutile.de

\title{
punkt-um.net
}

\section{Ihre Internet-Plattform zum Infodienst punkt. um für Informationen zu Nachhaltigkeit und Ökologie}

\author{
Aktuelle Termine - News aus dem Internet - Web-Site-Datenbank \\ OekoDok: Abstracts-Datenbank zu 60.000 Zeitschriftenartikeln seit 1987
}

Sie finden uns unter: http://www.punkt-um.net 
(c) 20I0 Authors; licensee IÖW and oekom verlag. This is an article distributed under the terms of the Creative Commons Attribution Non-Commercial No Derivates License (http://creativecommons.org/licenses/by-nc-nd/3.o/), which permits unrestricted use, distribution, and reproduction in any medium, provided the original work is properly cited. 